\title{
An heuristic uncertainty directed field sampling design for digital soil mapping
}

\author{
Shu-Jie Zhang a,e ${ }^{\text {, A-Xing Zhu }}{ }^{\text {b,c,d,a,*, Jing Liu }}{ }^{\text {d }}$, Lin Yang ${ }^{a}$, Cheng-Zhi Qin ${ }^{a}$, Yi-Ming An ${ }^{\text {a }}$ \\ a State Key Lab. of Resources and Environment Information System, Institute of Geographical Sciences and Resources Research, Chinese Academy of Sciences, Beijing 100101, China \\ b Jiangsu Center for Collaborative Innovation in Geographical Information Resource Development and Application, 1 Wenyuan Road, Nanjing, Jiangsu 210023, China \\ c Key Laboratory of Virtual Geographic Environment, Ministry of Education, Nanjing Normal University, 1 Wenyuan Road, Nanjing, Jiangsu 210023, China \\ d Department of Geography, University of Wisconsin, Madison, WI 53706, USA \\ e Policy Research Center for Environment and Economy, Ministry of Environmental Protection, Beijing 100101, China
}

\section{A R T I C L E I N F O}

\section{Article history:}

Received 24 December 2014

Received in revised form 3 December 2015

Accepted 13 December 2015

Available online 8 January 2016

\section{Keywords:}

Legacy samples

Individual representativeness

Prediction uncertainty

Stepwise sampling scheme

\begin{abstract}
A B S T R A C T
Legacy samples are a valuable data source for digital soil mapping. However, these sample sets are often small in size and ad hoc in spatial distribution. Constrained by the limited representativeness of such a sample set, the obtained soil maps are often incomplete in spatial coverage with "gaps" at the locations which cannot be well represented by these samples. The maps may also contain areas of high prediction uncertainty. In order to extend the predicted area and reduce prediction uncertainty, additional samples are needed. This paper presents a sampling design based on prediction uncertainty to select samples which will effectively complement the sparse and ad hoc samples, and maximize the spatial coverage of prediction and minimize prediction uncertainty. A case study in China shows that this sampling scheme was effective in achieving these goals. Compared with stratified random sampling scheme, when the number of additional samples is the same, the produced map using uncertainty directed samples has larger predicted area, and the accuracy of the produced map is higher than that of the maps using stratified random samples. The finding of this study suggests that prediction uncertainty is a useful indicator to aid field sample selection and to complement the legacy data. Furthermore, the mapping accuracy produced using this method can be quantitatively related to the number of additional samples needed which opens a new horizon for digital soil mapping.
\end{abstract}

(c) 2015 Published by Elsevier B.V.

\section{Introduction}

Legacy samples which were accumulated through historical national soil surveys and/or specific field studies are a valuable data source for digital soil mapping. However, in most areas especially in developing countries, the number of legacy samples could be so limited that it may not be appropriate to apply traditional methods (such as regression or kriging interpolation methods) to map soils using these samples. For example, the land area of China is about $9.6 \times 10^{6} \mathrm{~km}^{2}$, but there are only 7292 legacy profiles available across the country (about 1 sample per $1316 \mathrm{~km}^{2}$ in average) in the Soil Attributes Database from the Soil Series of China (volumes 1-6) and Soil Series of Provinces (total 34 volumes) (Yu et al., 2007; Shi et al., 2007). Some legacy samples were collected without following any conventional sampling designs (e.g. stratified random sampling, regular sampling). These samples, which are limited in number and do not provide a good spatial coverage of the study area, are referred to as sparse and ad hoc samples in this paper. Sparse and ad hoc samples are especially common for large

\footnotetext{
* Corresponding author at: School of Geography, Nanjing Normal University, No. 1, Wenyuan Road, Xianlin University District, Nanjing 210023, China.

E-mail address: azhu@wisc.edu (A.-X. Zhu).
}

area but cannot be used with the conventional soil mapping methods (such as regression or kriging) for mapping the spatial distribution of soil properties. Besides, it is difficult to collect a large set of samples following a well-defined sampling design due to restrictions in sampling under complex field conditions and limited sampling budget.

However, each of these sparse and ad hoc samples does contain the local relationship between soil and environmental conditions although these samples do not represent the entire area very well. To make full use of legacy samples, Zhu et al. (2015) presented an individual predictive soil mapping (iPSM) method to predict soil properties using sparse and ad hoc samples. Under the assumption that the more similar the environment conditions between two locations the more similar the soil property values (Hudson, 1992), they used similarity in environmental conditions between an unvisited location and a field sample to approximate the similarity between the soil at the unvisited location and that at the sample. Thus, the soil property value at the unvisited location can be computed based on the similarities to a set of samples and the property values at these samples (Zhu, 1997; Qi et al., 2007; Zhu et al. (2010a)). The method also produces uncertainty associated with each predication based on the similarities of the unvisited location to a set of samples. A "No Data" value will be assigned to locations where uncertainty values exceed a certain threshold (user-specified) under the 
notion that it is not reasonable to predict soil property values for locations which the current set of samples cannot represent well. Therefore, it is very possible to end up with an incomplete soil property map with areas of "No Data" values using this method and it is necessary to collect additional samples to cover these unmapped areas and to reduce the overall uncertainty of the predicted map.

The question then is how to effectively use the computed uncertainty information in designing a sampling scheme which can integrate existing samples and provide as few samples as possible but will maximize the reduction of the unmapped areas and minimize the overall uncertainty in the predicted map.

Design-based sampling (regular grids, simple random sampling, etc.) is very difficult to integrate legacy samples, because the legacy samples are always ad hoc (Brus and de Gruijter, 1997; Walvoort et al., 2010). For model-based sampling scheme, the semi-variance function which quantifies the spatial auto-correlation is always estimated from a large amount of existing samples (Brus et al., 2006; Isaaks and Srivastava, 1989; Goovaerts, 1999). But, the legacy samples are usually sparse, which makes the model-based sampling scheme not suitable for integrating legacy samples.

The conditioned Latin hypercube sampling (cLHS) as proposed by Minasny and McBratney (2006) has the advantage that the distribution of the designed sample locations replicates the distribution of environmental covariates. cLHS is used widely at present (Worsham et al., 2012; Taghizadeh-Mehrjardi et al., 2014; Reza Pahlavan Rada et al., 2014; Kidd et al., 2015). But each cLHS sampling scheme is designed independently, thus cLHS is hardly used for designing additional sample and it cannot provide the sampling order of the additional samples.

Spatial Simulated Annealing (SSA) method could be used to optimize placement of the individual observations by meeting some criterion (the minimal average or maximum Kriging variance), and this optimization sampling method also could include use of previous samples to direct additional sampling (van Groenigen et al., 1999; Van Groenigen, 2000; Brus and Heuvelink, 2007). However, SSA method cannot provide the sampling order of the additional samples also. When the sampling resource is limited, sampling order is very important information for allowing investigators to effectively plan sampling resources.

Purposive sampling intends to collect samples which are typical of soil types or soil mapping units. Purposive samples are usually designed by local soil experts based on their knowledge during conventional soil mapping. But this type of purposive sampling highly depends on experience or personal judgment of soil experts. An integrative hierarchical stepwise sampling strategy has been proposed to design representative samples with assistance of environmental covariates through a fuzzy clustering approach (Yang et al., 2013). Although this method is effective for predicting soil maps in digital soil mapping for initial sampling, this method has not been used in additional sampling, and it also does not have any mechanism to include uncertainty in the design.

This paper presents an effective and stepwise sampling scheme to identify additional sample locations based on the prediction uncertainty information quantified by the iPSM method proposed by Zhu et al. (2015). The method not only effectively extends the mapped area and reduce overall uncertainty using as few samples as possible, but also integrate all the legacy soil samples. Section 2 presents the details of this uncertainty directed and stepwise sampling scheme, which is followed by a case study illustrating the effectiveness of the proposed method. Section 4 presents the result and discussion. Conclusions are drawn in Section 5.

\section{Methods}

\subsection{Basic idea}

The basic idea of the uncertainty directed sampling reported in this paper is to use the uncertainty derived from the method (iPSM) by Zhu et al. (2015) to identify as few samples as possible to map soil spatial distribution below a user specified level of prediction uncertainty and to maximize the reduction of the prediction uncertainty.

Zhu et al. (2015) proposed the individual predictive soil mapping (iPSM) method which can make full use of limited soil sample data for predictive soil mapping and provide the prediction uncertainty at every location where a prediction is made. iPSM uses the soil-environment relationship at each individual soil sample location to predict soil properties at unvisited locations and estimate prediction uncertainty. The more similar the soil environmental conditions between an unvisited location and the locations of legacy soil samples, the lower the prediction uncertainty on the unvisited location because the legacy data could represent the unvisited location well. Prediction uncertainty can be measured before a prediction is made for a given location and a given set of soil sample locations. Thus, with iPSM a user can specify a level of prediction uncertainty under which the soil property at an unvisited location can be predicted. Locations with uncertainty values higher than the specified level are assigned "No Data" to signify that the current set of samples is insufficient to make soil property estimation at the given level of acceptability (as specified by the uncertainty level) (Zhu et al., 2015). These locations form holes or gaps on the output soil property map. Fig. 4 shows the map with unmapped area (gray area) which cannot be represented by legacy samples well. The uncertainty on the gray area is higher than a specified threshold, and "No Data" would be assigned to these locations when mapping soil property.

To examine the effects of sampling on gap filling and on uncertainty reduction, the proposed method were divided into two stages. The first is "Gap filling", which is to identify as few samples as possible to complete the prediction for areas previously labeled as "No Data". The second is to select samples which can maximize the reduction of overall prediction uncertainty. To fill the gaps of soil map and at the same time to minimize the number of samples needed, we adopt the following process: 1) in the unmapped region ("No Data" data), the location which can extend the predicted area most is chosen as the first additional sample and add this sample into the pool of existing samples; 2) we update the soil map based on the existing samples (including the newly selected additional sample); 3 ) if the resulted soil map still contains "No Data" locations or the "No Data" areas are still too large to be acceptable, select the location which has most incremental predicted area as the second additional sample. Repeat this process till the soil map is complete or the "No Data" areas are below an acceptable level (user-specified).

To maximize the reduction of the overall prediction uncertainty we adopt the following process: 1 ) based on the assumption that the more similar the environment conditions between two locations the more similar the soil property values, we select the additional sample representing the largest area in high uncertainty region and add this sample into the pool of existing samples; 2) update the prediction uncertainty map using the existing samples (including the additional sample just selected). Repeat the above process till the overall uncertainty is below a certainty threshold or the sampling is beyond the project budget. These additional samples for reducing the overall uncertainty are also stepwise because each sample is based on the uncertainty map generated from the last round.

The above uncertainty directed sampling method not only integrates the legacy samples but also provides as few stepwise samples as needed to fill the map gaps and reduce the overall prediction uncertainty. In addition, at the time of selecting a sample location using this design does not mean actually filed sampling at this location, because the selection for maximization of area covered or minimization of uncertainty is based on environmental similarity only. Calculation of environment similarity does not need the soil property value at the sample. In fact, the user(s) can wait till the locations of all the additional samples (based on the budget and or area coverage and or uncertainty reduction defined by users) are determined through the above process before starting the field sampling campaign so that samples can be collected at once in the field. 


\subsection{Uncertainty directed sampling design}

As outlined in Section 2.1, the whole process of the uncertainty directed sampling scheme consists of two stages: 1 ) to efficiently locate new samples, as few as possible to complement the legacy sample set in order to fill the "No Data" gaps and extend predicted area, 2) to add as few new samples as possible to improve prediction accuracy by reducing the overall uncertainty of prediction. For each of the two stages, prediction uncertainty needs to be defined first. Fig. 1 shows the flowchart of overall methodology.

\subsubsection{Determination of prediction uncertainty}

The determination of prediction uncertainty contains 3 steps: a) selection of covariates; b) calculation of similarities between unvisited locations and samples; and c) determination of prediction uncertainty.
2.2.1.1. Selection of covariates. Selection of appropriate environment covariates is the first and very important step for calculating the similarity between two locations. The key to selecting environmental covariates is that the covariate reflects the spatial variation of soils and at the same time data on spatial variation of this covariate can be easily obtained. With the development of earth observation technologies and spatial analysis method, many new useful covariates are emerging (Nield et al., 2007; Zhu et al. (2010b); Liu et al., 2012; Qin et al., 2012). Statistical methods (such as principal components analysis, stepwise regression) could help us select appropriate covariates (Pechenizkiy et al., 2003; Behrens et al., 2010; Samuel-Rose et al., 2015; Ramadan et al., 2001; Mansuy et al., 2014; Beaudoin et al., 2014). On the other hand, covariates could also be selected based on expert knowledge or related previous studies in the same study area. The soil property is the result of climate condition, parent material, topographical factors and time.

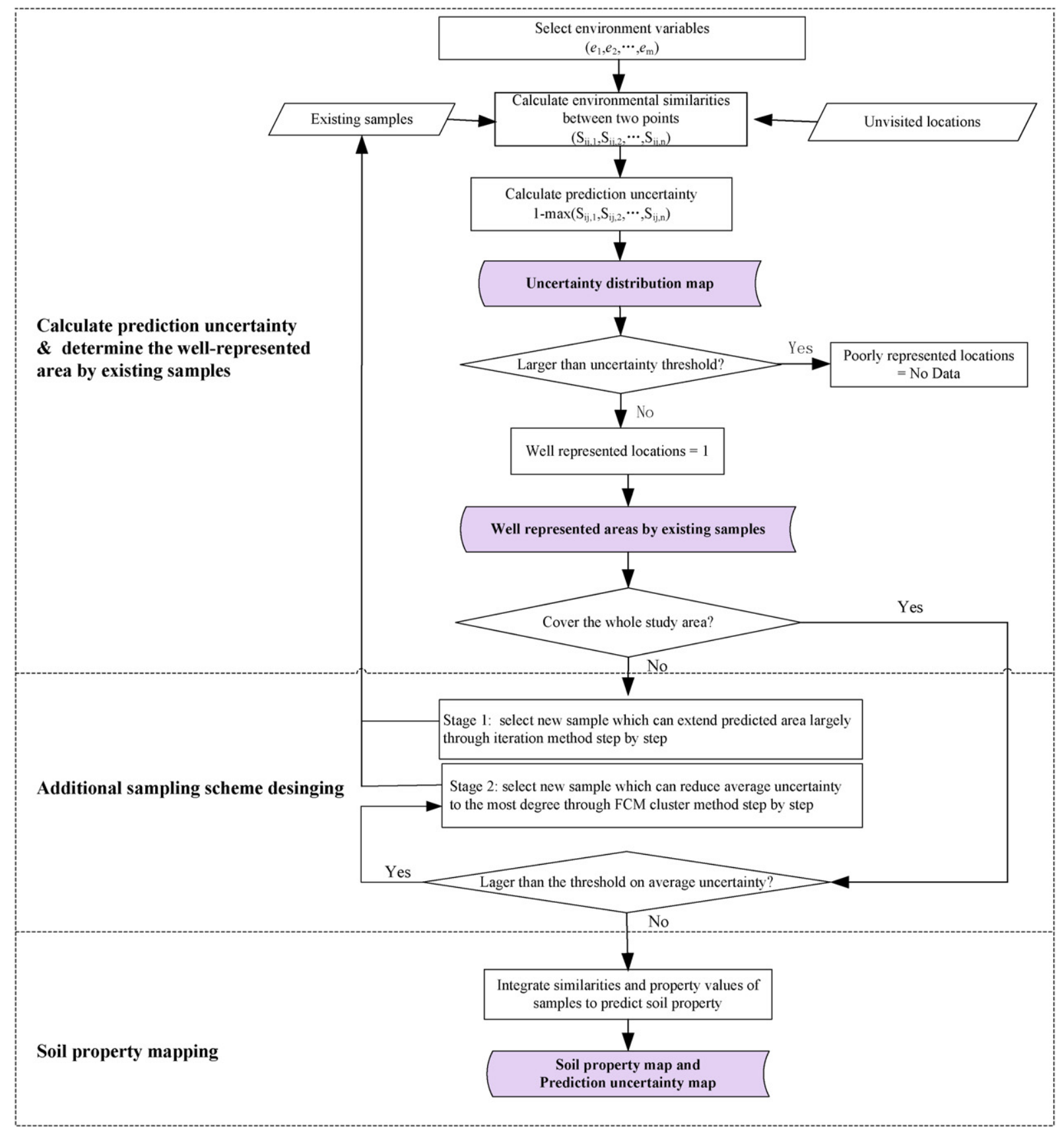

Fig. 1. The overall flowchart of uncertainty directed sampling scheme. 
The Soil-landscape Model "CLORPT" (Jenny, 1941) and the SCORPAN model (McBratney et al., 2003) list lots of possible relating covariates.

2.2.1.2. Calculation of similarities. Similarity calculation was carried out on two levels: individual environment covariate level and location level by integrating all environmental similarities, which was demonstrated as Eq. (1) (Zhu et al., 1996; Zhu et al., 2015).

$S_{i}^{k}=\stackrel{m}{P}_{v=1}\left(E^{v}\left(e_{i}^{v}, e_{k}^{v}\right)\right)$

in which $S_{i}^{k}$ represents the similarity between a location (i) and sample $k$; $e_{i}^{v}$ is the value of the environment covariate $v$ on location $(i) ; e_{k}^{v}$ is the value of the covariate $v$ associated with sample $k ; m$ is the number of covariates.

$E()$ is the function for calculating similarity at individual environmental covariate level. The $E$ function is associated with the data type of environment covariates. For example, when the data type of covariate is in nominal or ordinal type (e.g. parent material), $E$ is usually a Boolean function; when the data type of covariate is in interval or ratio type (e.g. temperature, slope), $E$ may be Euclidean distance, Mahalanobis distance, Gower distance, etc. (Shi et al., 2005). In this paper, we adopted Boolean function and Gower distance to calculate individual variable similarity.

$P()$ is the function for calculating similarity at location level. In this process, we consider the relationship not only between environment covariates distribution and soil property distribution but also between different environment covariates. The limiting factor approach was adopted to integrate the similarities on individual environment variable level that is the smallest value among all covariates similarities was used as the environmental similarity at location level (Zhu and Band, 1994).

Based on Eq. (1), the similarities of each location to all samples could be calculated, which can form an "environment similarities vector" at each location as is shown in Eq. (2).

$\vec{S}_{i}=\left(S_{i}^{1}, S_{i}^{2}, \ldots, S_{i}^{n}\right)$

in which, $\vec{S}_{i}$ is the environment similarities vector at location $(i) ; S_{i}^{k}(k=$ $1,2, \cdots, n)$ is the environment similarity between the location $(i)$ and sample $k ; n$ is the number of samples.

2.2.1.3. Determination of prediction uncertainty. The "environment similarities vector" indicates the degree how well the sample set can represent the location. Prediction uncertainty could be quantified by analyzing this environment similarity vector. In this paper, prediction uncertainty at each location was calculated based on Eq. (3)

Unertainty $_{i}=1-\max \left(\vec{S}_{i}\right)=1-\max \left(S_{i}^{1}, S_{i}^{2}, \ldots, S_{i}^{n}\right)$

This equation means that if the maximum value of all the environmental similarities of location ( $i$ ) to all samples is low, then the existing samples cannot represent the unvisited location $(i)$ well and the prediction uncertainty will be high. This uncertainty calculation method has been proved to be effective in other previous study (Zhu, 1997; Zhu et al., 2015). According to the previous research, the uncertainty was positively related to prediction residuals and can be used as an indicator of its prediction accuracy. It must be noted that this uncertainty describes the uncertainty involved in predicting the soil property value at a location which cannot be well-represented by the existing set of samples. It does not capture the uncertainty due to the errors in the data describing the environmental variables involved in defining the similarity.

\subsubsection{Gap filling stage}

In this stage, the objective is to select a minimum number of samples to fill the "No Data" gaps. A method of combining Traversing algorithm and Greedy algorithm was adopted and it is described as follows:

(1) For a given uncertainty threshold defining the areas of "No Data", each location in the "No Data" regions is treated as a candidate sample. First, calculate the similarities between a candidate sample and all the other locations with "No Data" based on Eqs. (1) and (2). Then, quantify the prediction uncertainties on all the locations with "No Data" based on Eq. (3).

(2) When the uncertainty on a "No Data" location is lower than the given uncertainty threshold, the location could be represented by the candidate sample. Therefore, the incremental predicted area of the candidate sample can be calculated by counting the number of locations which were previously "No Data" but with the uncertainty lower than the above given threshold if the candidate sample was selected. Finally, compare the incremental predicted areas of all candidate samples and the candidate sample which can extend predicted area most will be chosen as the additional sample for this round.

(3) Add the new sample to the existing sample set to form a new sample set and update the uncertainty map as well as the predicted area map based on this renewed sample set using the method of Zhu et al. (2015).

Iterate the above three steps until the predicted area covers the whole study area under the current threshold or until the total size of unmapped areas is below a user defined value.

\subsubsection{Uncertainty reduction stage}

The objective of this stage is to design a minimum number of samples to reduce the overall prediction uncertainty. The procedure of uncertainty reduction stage is shown as Fig. 2.

(1) Select the stratum which contains the largest area with high uncertainty.

Produce an uncertainty map using the sample set obtained in Section 2.2.2. Set a "high uncertainty" threshold, any value over which the prediction is considered with high uncertainty. Different from the uncertainty threshold which is used to determine "No Data" area, this threshold is used to decide what area is predicted with "high uncertainty" and used for uncertainty reduction. Divide the areas into two parts using this threshold: one with uncertainty higher than the threshold and the other with uncertainty lower than the threshold.

For large areas, categorical environmental variables such as parent material, land use type, are always chosen as important covariates. In this case, tabulate the size of uncertainty area over each stratum and then select the stratum with the largest high uncertainty area. If no categorical covariates area involved, then go to step (2) directly.

(2) Identify the environment class that has the largest area of high uncertainty values in the selected stratum.

For the selected stratum from (1), a fuzzy c-means classification (FCM) technique is implemented to obtain the environment clusters over the high uncertainty areas. Select the environmental class with the largest number of pixels as the candidate group from which to draw the sample.

(3) Find the candidate sample locations.

The probability density function (PDF) of the prediction uncertainties in the largest environment condition class obtained from (2) is estimated and the modal value of the PDF is determined to find the candidate sample locations. The reason that we used PDF but not histogram is that PDF is smoother and more continuous. Compared with the simple histogram, PDF can describe the distribution of data better, so it was used to analyze the distribution if prediction uncertainty. In this paper, probability density function (PDF) was estimated by Kernel Density Estimation method (KDE as Eq. (4)) (Silverman, 1986) 


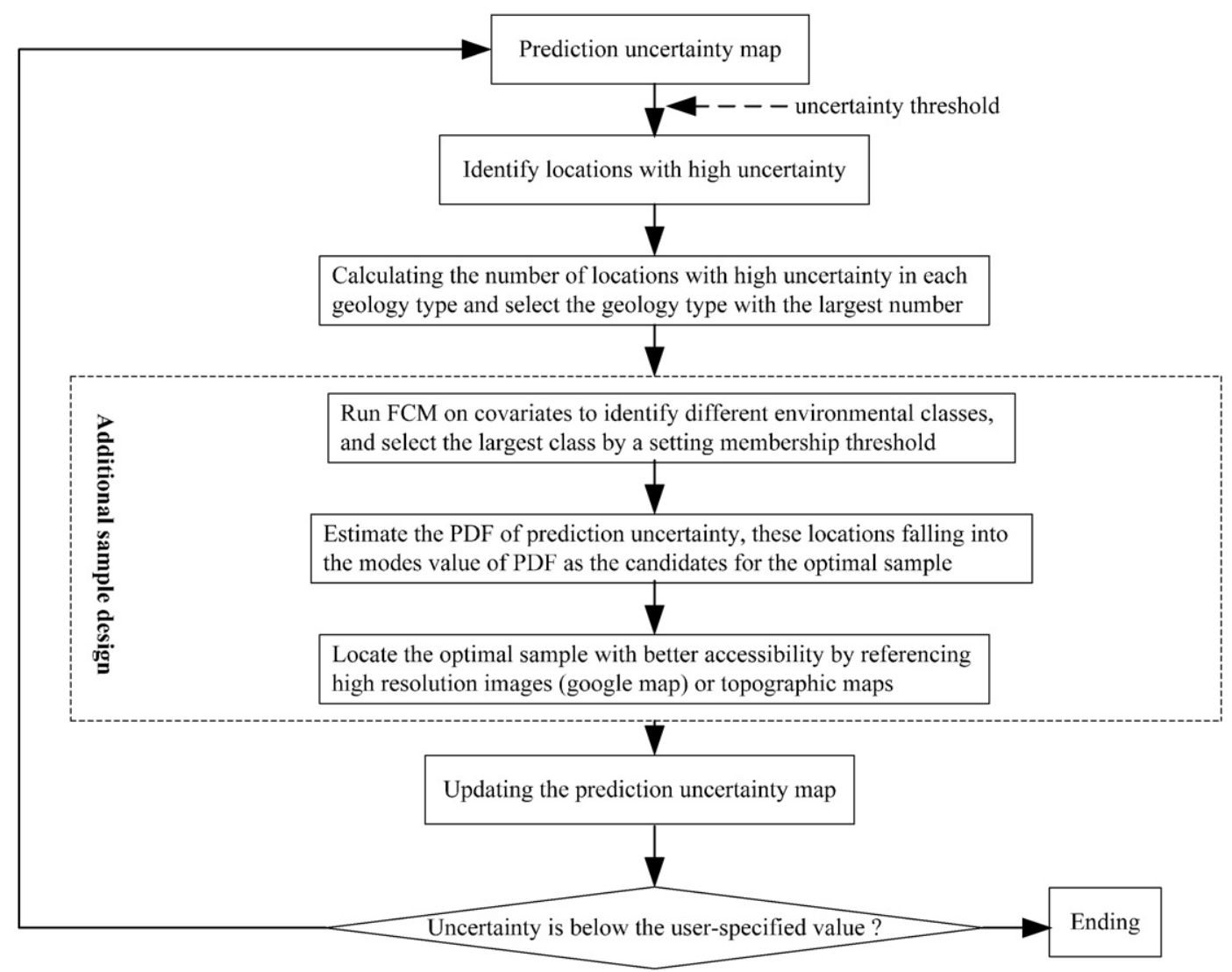

Fig. 2. The flowchart of the uncertainty reduction stage.

to select the sample.

$f_{h}(x)=\frac{1}{N h} \sum_{i=1}^{N} K\left(\frac{x-x_{i}}{h}\right)$

in which $f_{h}(x)$ is the PDF of prediction uncertainty $x_{i}(i=1,2, \cdots, N), K()$ is a kind of kernel function and it is Gaussian kernel in this paper, $h$ is bandwidth which influences the smoothing degree of PDF curve and it is determined using "rule of thumb" method (Sheather and Jones, 1991).

The locations with the uncertainty falling into the range enclosing the mode of PDF should represent the largest area with high uncertainty if these locations are selected as additional samples, and these locations are more effective in reducing the overall uncertainty. The width of modes of the PDF is defined between the maximum value of the PDF minus the bandwidth $h$ and the maximum value of PDF plus the bandwidth $h$.

(4) Locate the optimal sample

First, the optimal sample will be located on the area with candidate samples clustering. Then, select the location with better accessibility as the optimal sample by referencing high resolution remote sensing images (Google map) or topographic maps.

(5)Add the new sample to the existing sample set, then update the uncertainty map based on the renew sample set and calculate the overall uncertainty level over the entire area.

Iterating procedures (1) through (5) until the overall prediction uncertainty drops below the user-specified value or the number of the samples is larger than the number planned.

\subsection{Validation}

In order to compare the effectiveness, we will compare uncertainty directed sampling scheme with stratified random sample scheme in two aspects: the incremental predicted area and the accuracy of soil map by adding one sample at a time. RMSE will be calculated for validating soil map accuracy. So, two other sample sets need to be collected with one based on the regular sampling strategy (referred to as the regular sample set) and the other based on the stratified random sampling strategy (referred to as the stratified random set). The stratified random set will be designed as follows: First, the entire study area is stratified based on categorical covariate or other criterion (such as clusters generated some covariates). The number of samples in each stratum depends on the area proportion of the stratum in the entire study area. Second, design random samples for each stratum. For one stratum, random samples were located using a random generator in ArcGIS software. In this procedure, if the spatial distribution of the random samples is centralized, then we dropped all the samples in this stratum area and produced new random samples repeatedly until the random samples seem to disperse although this is not necessary. Third, combine the random samples in each stratum to form the stratified random sample set. Therefore, the stratified random samples in this paper should capture the global distribution characteristic of soil property.

In order to validate the stratified random sampling scheme comprehensively, the comparison between the results based on the samples from the uncertainty directed design with these based on samples from stratified random design is done using a sample-wise approach. In other words, we want to see the effects between the two sampling strategies by adding one sample at a time. The samples from the uncertainty directed design are already ordered based on their respective impact on the reduction of unmapped area and uncertainty. The samples from the stratified random samples do not have order in similar nature. But which sample from the stratified random samples to be added next would have an important impact on the inference outcome. To minimize this impact we created 5 ordered sets of samples from the stratified random samples through randomly selecting a sample from the pool of stratified random samples for each set. The comparison is then 
done by comparing the results based on the samples from the uncertainty directed sampling with each of the 5 sets of ordered samples from the stratified random samples.

\section{Case study}

\subsection{Study area and legacy samples}

The study area is located between $48^{\circ} 53^{\prime} 24^{\prime \prime}-48^{\circ} 59^{\prime} 24^{\prime \prime} \mathrm{N}, 125^{\circ} 8^{\prime}$ $24^{\prime \prime}-125^{\circ} 16^{\prime} 12^{\prime \prime} \mathrm{E}$, which includes Xuan-Zhou county, Lang-Xi county and Guang-De county in south of Anhui province, China (Fig. 3). It is about $110 \mathrm{~km}$ by $84 \mathrm{~km}$ covering $5900 \mathrm{~km}^{2}$ with a highest altitude of $1039 \mathrm{~m}$. This area belongs to a transition zone between the middle and lower reaches of Yangtze River and mountainous area of southern Anhui.

The area is under a subtropical monsoon climate. It is hot and rainy in summer because of ironical maritime air and cold and dry in winter resulting from Eurasia air-mass. Annual average temperature varies from $11.6{ }^{\circ} \mathrm{C}$ to $15.8^{\circ} \mathrm{C}$ and annual average precipitation is $1240 \mathrm{~mm}$ to $1780 \mathrm{~mm}$. The parent materials are mainly composed of mild clay-silt-gravel formed during Quaternary system and sandstone with proportions of $34 \%$ and $15 \%$, respectively. The landform is characterized by hill and plain with some mountains in the south. The characteristic vegetation types are evergreen coniferous forest and deciduous broad-leaved forest, most of which are secondary forests or human-made forests. Most of land use types in this area are farmland and the stable crop is rice. The whole study area experiences severe human activities. Soil types are mainly red soil and paddy soil, accounting for $46.5 \%$ and $39.7 \%$, respectively, according to the 1:100,000 Chinese Soil Map.

There are only 6 legacy soil profiles from the Second National Soil Survey of China. It is obvious that the size of legacy samples is too limited to use the existing predictive soil mapping methods for soil mapping in this area. Therefore, the individual predictive soil mapping (iPSM) method is appropriate to predictively map the soil property over this area (Zhu et al., 2015). Fig. 3 shows the locations of the legacy sampling points.

\subsection{Selection of environmental covariates}

Based on the understanding of the soil-environment relationship and previous work in this study area (Zhao et al., 2007; Sun et al., 2008; Yang et al., in press), we selected environment covariates on three aspects including climate, parent material and topography. Climate covariates include annual average precipitation and annual average temperature with $1 \mathrm{~km}$ resolution, which were provided by Laboratory of Ecosystem Network Observation and Modeling, Institute of Geographic Sciences and Natural Resources Research (IGSNRR), CAS. These climate data were produced by interpolating observation point data at 740 weather stations during the period of 1971-2000 in China (Feng et al., 2007; Yu et al., 2004) Geology Type Map was used to describe the parent material, the scale of which is 1:500,000 and they were provided by Institute of Soil Science, CAS. We understand that the spatial resolution (or scale) of the geology data layer is very coarse but it is the only geological data available for information on parent materials over this large area. The 27 different rock types (referred to geology type from hereon) in the geology map were grouped into 8 types based on the characteristics of lithology. Topographic covariates include slope, topographic wetness index (TWI), plan curvature and profile curvature which were all derived from DEM with $90 \mathrm{~m}$ resolution (http://glcf.umiacs.umd.edu/). Slope, plan curvature and profile curvature were generated using the 3dMapper software (http://www. TerrainAnalytics.com). TWI was calculated using a multiple-flowdirection algorithm (Qin et al., 2007). Annual average precipitation, annual average temperature and the geology type data was resampled into $90 \mathrm{~m}$ resolution by duplicating the value of the $1 \mathrm{~km}$ at $90 \mathrm{~m}$ level to match the DEM resolution. We understand that this "downscaling" may be acceptable for annual average precipitation and annual average temperature, but may not be appropriate for the geology type data due to the fact that geology information may not be uniform over the $1 \mathrm{~km}$ by $1 \mathrm{~km}$ area but this is the best data we have.

\subsection{Determination of the unmapped area}

The uncertainty from individual predictive soil mapping method (Zhu et al., 2015) is a measurement of how reliable it is to use existing
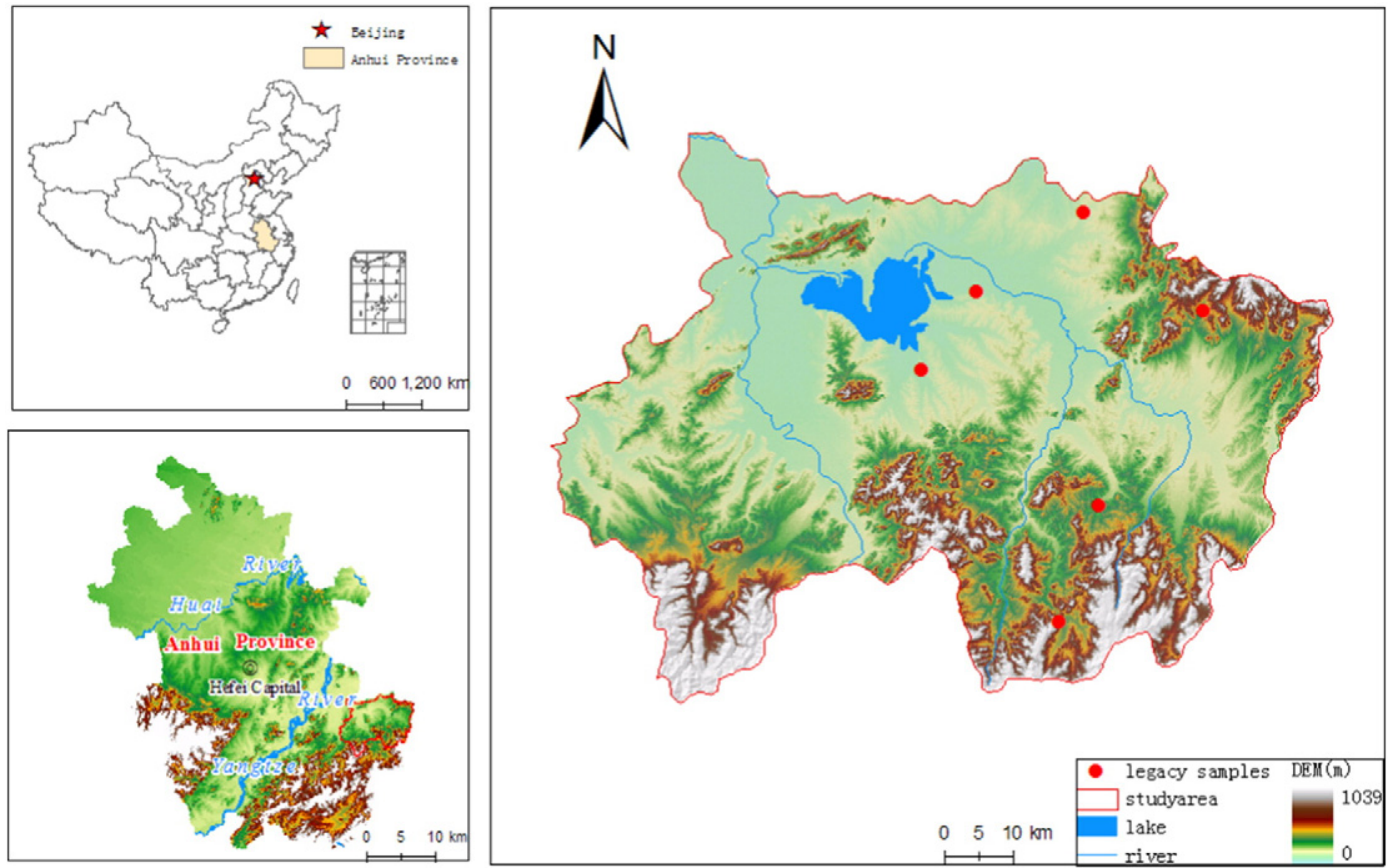

Fig. 3. Study area and legacy soil samples. 
soil samples to represent a given unvisited location for prediction. The more similar the environment similarities between an unvisited location and existing samples, the lower the prediction uncertainty on the unvisited location. The choice of uncertainty threshold depends on the user's tolerance regarding the reliability of using existing soil samples to represent the unvisited location. The maximum value for prediction uncertainty is 1 . When the uncertainty was smaller than 0.5 , in other words, the environment similarities between the unvisited location and all the samples is bigger than 0.5 , we think the unvisited location could be represented by the existing samples in this paper. Otherwise, the unvisited location could not be represented and it is unacceptable to predict the soil property on the unvisited location based on existing samples. So, we chose 0.4 as the uncertainty threshold. Set the uncertainty threshold as 0.4 , reclassify the area as "No Data" where the prediction uncertainty is higher than 0.4. Fig. 4 shows the unmapped area ("No Data" area) based on soil mapping using the 6 legacy samples.

\subsection{Gaps filling stage}

Fig. 4 shows that there is large gray area with "No Data" using the 6 legacy samples to map the soils when the uncertainty threshold is 0.4 . Therefore, it is necessary to design additional samples to extend the predicted area (in green) to the entire study area. The steps in selecting addition samples are described below. This is done for one sample at a time.

(1) Each location in the "No Data" area can be seen as a candidate sample. Calculate the uncertainty by analyzing the environmental similarities between the candidate sample and all other locations in the "No Data" regions.

(2) For given the uncertainty threshold 0.4, compare the incremental predicted area of each candidate sample. The candidate sample which can extend the predicted area most was chosen as the additional sample for this round.

(3) Add the additional sample to the existing sample set (the legacy sample set if the new sample is the first additional sample) and update the prediction uncertainty map and the predicted area map based on the renewed sample set.
Iterate the above procedure until the mapped area covers the whole study area or the total unmapped area gets below a user specified value. Finally, organize the optimal additional samples in the order it was selected (Table 1).

In our study area, there are 687,887 grids (90 $\mathrm{m} * 90 \mathrm{~m}$ ) in total. When 50 additional samples were added to the 6 legacy samples the predicted area would cover the entire study area. However, starting from the 26th sample the incremental predicted area (grids) is less than 100 grids $\left(0.81 \mathrm{~km}^{2}\right)$. So it is not worth costing much labor and financial resource to sample the last 25 samples. In this paper, we only choose the first 25 samples listed in Table 1. Fig. 5 showed the distribution map of the selected 25 samples. From Fig. 5 we can see that most of the additional samples which designed in this gap filling stage are in the southern mountain area. Because of the more complex terrain environment, it is reasonable that samples have less individual representativeness and more samples were needed to represent the whole mountain area.

\subsection{Uncertainty reduction stage}

After the additional samples designed in the gap filling stage were added to legacy samples the new sample set can well represent almost the entire study area given the uncertainty threshold. The next objective is to select as few samples as possible to reduce the overall uncertainty so as to improve the accuracy of soil property map.

In order to describe the process clearly, we took the selection of the first sample as an illustration.

(1) Select the geology type stratum with largest number of high uncertainty locations.

First, add the 25 additional samples designed in the filling gap stage to the legacy sample set, and then produce a prediction uncertainty map based on the above sample set using individual predictive soil mapping method (Zhu et al., 2015). Second, set the "high uncertainty" threshold to be 0.3 . In this study area there are 8 geology types and the number of locations with uncertainty higher than 0.3 in each geology class is shown in Table 2. Based on this table, the first sample in the uncertainty reduction stage should be selected from the area covered by mild claysilt-gravel geology formed during Quaternary system, Geology Type 6.

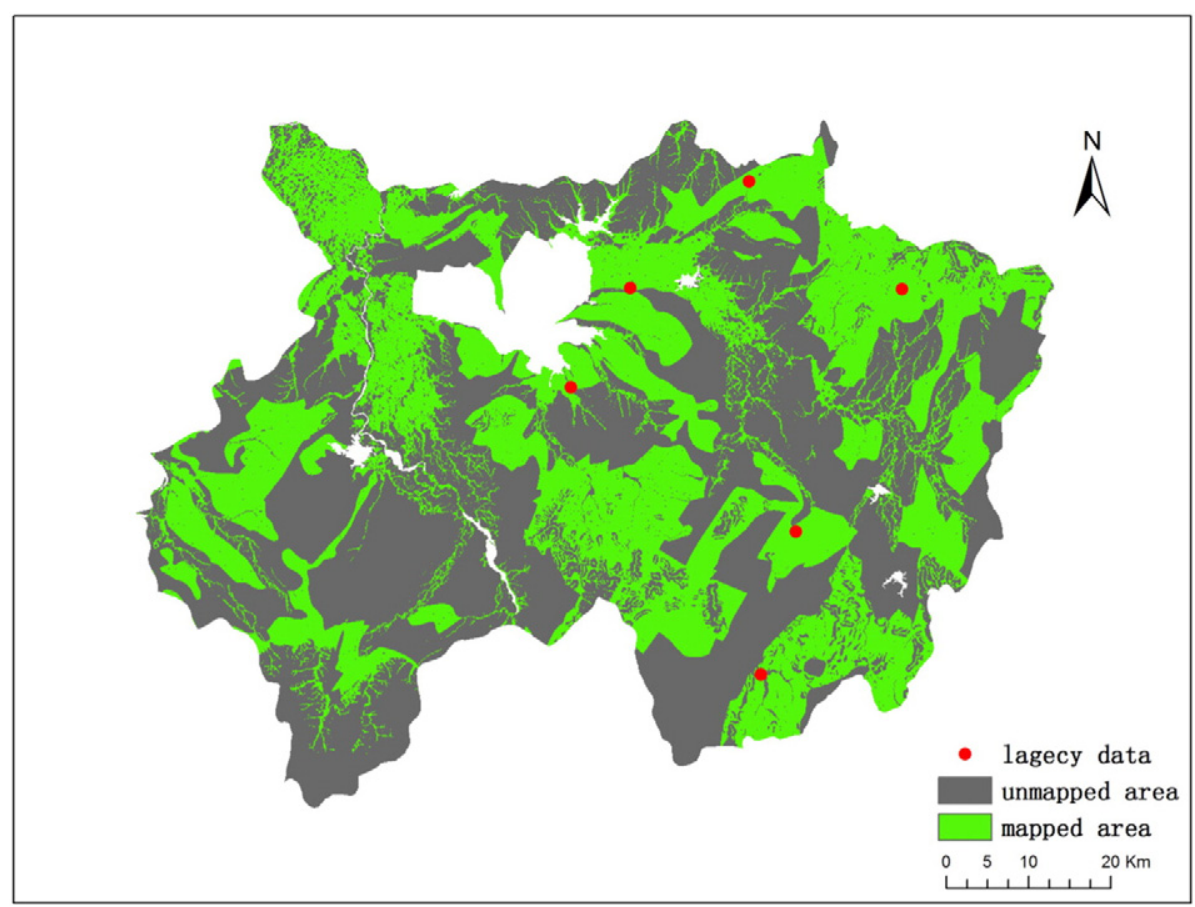

Fig. 4. The spatial distribution of unmapped area (property with "No Data"). 
Table 1

The incremental predicted area of additional samples designed in the gap filling stage.

\begin{tabular}{cclcll}
\hline & $\begin{array}{l}\text { Incremental } \\
\text { Order } \\
\text { predicted area } \\
\text { (number of grids) }\end{array}$ & $\begin{array}{l}\text { Incremental } \\
\text { predicted } \\
\text { area/total } \\
\text { grids(\%) }\end{array}$ & Order & $\begin{array}{l}\text { Incremental } \\
\text { predicted area } \\
\text { (number of grids) }\end{array}$ & $\begin{array}{l}\text { Incremental } \\
\text { predicted } \\
\text { area/total } \\
\text { grids(\%) }\end{array}$ \\
\hline 1 & 153,211 & 22.27 & 26 & 75 & 0.01 \\
2 & 91,014 & 13.23 & 27 & 61 & 0.01 \\
3 & 45,330 & 6.59 & 28 & 60 & 0.01 \\
4 & 36,056 & 5.24 & 29 & 54 & 0.01 \\
5 & 23,980 & 3.49 & 30 & 46 & 0.01 \\
6 & 13,878 & 2.02 & 31 & 33 & 0.005 \\
7 & 7191 & 1.05 & 32 & 32 & 0.005 \\
8 & 1411 & 0.21 & 33 & 26 & 0.004 \\
9 & 1116 & 0.16 & 34 & 16 & 0.002 \\
10 & 1085 & 0.16 & 35 & 15 & 0.002 \\
11 & 650 & 0.09 & 36 & 14 & 0.002 \\
12 & 582 & 0.08 & 37 & 10 & 0.001 \\
13 & 441 & 0.06 & 38 & 9 & 0.001 \\
14 & 365 & 0.05 & 39 & 5 & 0.001 \\
15 & 312 & 0.05 & 40 & 5 & 0.001 \\
16 & 283 & 0.04 & 41 & 3 & 0.0004 \\
17 & 208 & 0.03 & 42 & 3 & 0.0004 \\
18 & 198 & 0.03 & 43 & 2 & 0.0003 \\
19 & 183 & 0.03 & 44 & 2 & 0.0003 \\
20 & 171 & 0.02 & 45 & 1 & 0.0001 \\
21 & 130 & 0.02 & 46 & 1 & 0.0001 \\
22 & 119 & 0.02 & 47 & 1 & 0.0001 \\
23 & 119 & 0.02 & 48 & 1 & 0.0001 \\
24 & 118 & 0.02 & 49 & 1 & 0001 \\
25 & 106 & 0.02 & 50 & 1 & \\
\hline & & & & & \\
\hline
\end{tabular}

(2) Select the largest environment class in Geology Type 6.

Run FCM on covariates (minus the geology type) for the location with high uncertainty in Geology Type 6 to identify different environment classes. Based on the coefficient (F) and entropy (H) (Zhu et al., 2008; Yang et al., 2011), the best cluster number is 5 and the fuzzy parameter $m$ is 1.5 . After hardening the cluster results, Class 1 has 40,135 cells and Class 2 through Class 5 have 20,875, 26,084, 17,585 and 11,426 cells, respectively. The largest environment class is Class 1 .

(3) Estimate the probability density distribution of the uncertainty in the environmental class 1 and identify the first optimal sample.
Table 2

The number of locations with uncertainty higher than 0.3 in each of geology types.

\begin{tabular}{llc}
\hline ID & Parent material & $\begin{array}{c}\text { Number of cells with } \\
\text { uncertainty }>0.3\end{array}$ \\
\hline 1 & Granite; diorite-porphyrite & 2070 \\
2 & Tuffaceous sandstone & 2288 \\
3 & Conglomerate & 10,493 \\
4 & Sandstone & 8425 \\
5 & Limestone & 11,592 \\
6 & Mild clay-silt-gravel formed during Quaternary system & 140,786 \\
7 & Glacial deposits & 951 \\
8 & Shale & 3668 \\
\hline
\end{tabular}

Fig. 6 shows the probability density distribution of the uncertainty in the largest environmental condition (Class 1). For a PDF with a signal peak, we locate the new additional sample point at the locations (candidate samples) with their uncertainty values closed to the mode of the probability density distribution of the uncertainty. For a PDF with two or more peaks, when the heights of the peaks are more or less the same, the new additional sample point would be designed at the locations with their uncertainty values closed to the last peak of the PDF. For a PDF with two or more peaks and the height of the peaks are different, the new additional sample point would be designed at the locations with their uncertainty values closed to the highest peak of the PDF. The selection of this sample would reduce the overall uncertainty the most.

All the candidate samples (the blue locations) for the first additional sample were shown in Fig. 7. In order to ensure accessibility to the sample, the optimal sample was selected by referring to topography map, land use map, and Google map.

(4) Add the additional sample to the existing sample set and iterate procedures (1) through (3) to select other optimal samples for uncertainty reduction.

Iterate procedures (1) through (3) till one of the following two criteria is met, then reduce the "high uncertainty" threshold 0.3 to 0.2 : (a) the largest number of locations with uncertainty higher than 0.3 is less than a user specified value in each geology type. For this paper we set this number to be 1000 . It is not worth to design a sample when the high uncertainty area is small; (b) when adding an additional

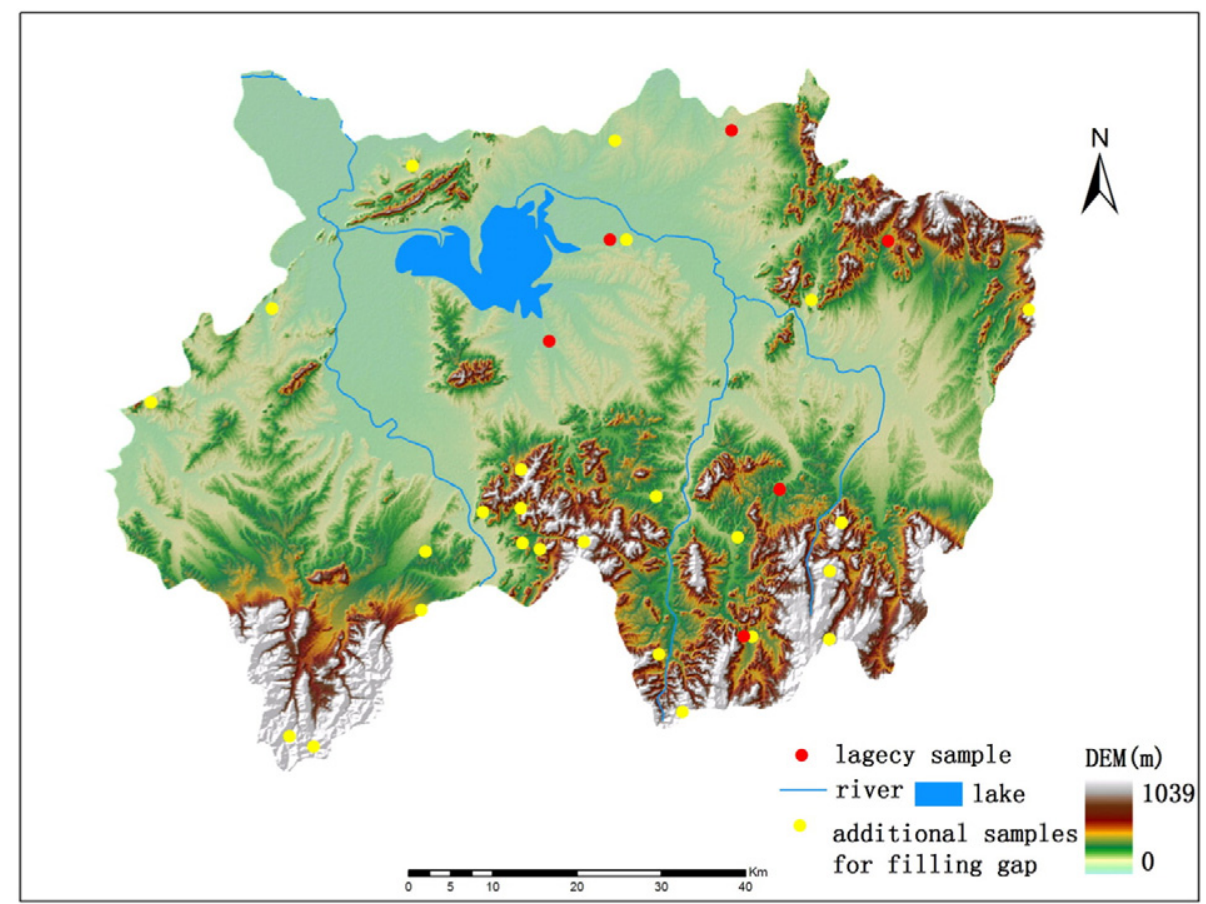

Fig. 5. The spatial distribution of additional samples for filling gap. 


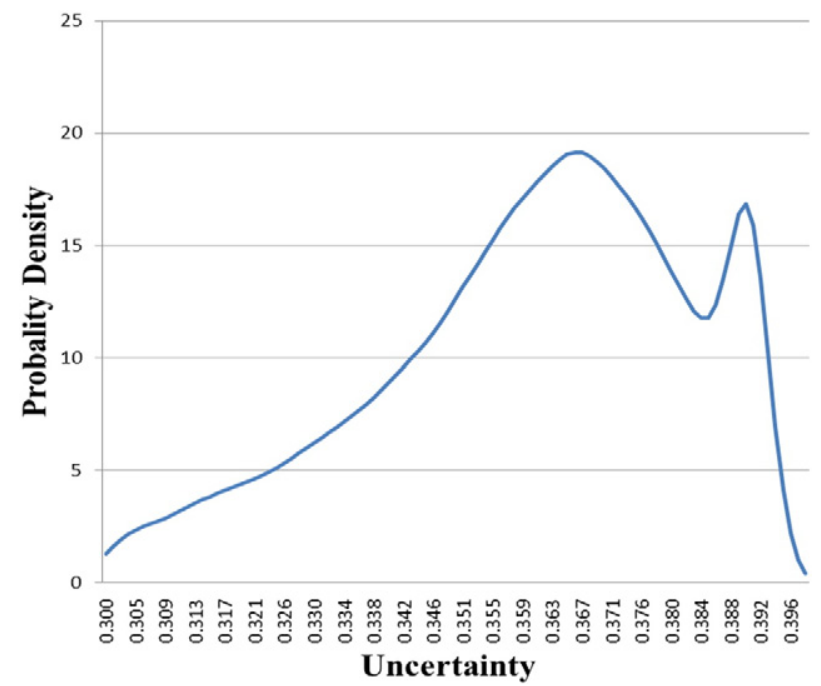

Fig. 6. PDF of the uncertainty in the largest environmental condition class 1.

sample the sum of the reduced uncertainty is less than a user specified number (set to 500 in this study) which means that the effect of adding a new sample is low.

In this paper, according to the above two criteria, the uncertainty threshold was reduced step by step till it is 0.1 . The choice of the uncertainty threshold depends on the user's tolerance reliability of using existing soil samples to represent the unvisited location and the sampling budget. In this paper, such a strict uncertainty threshold 0.1 means that the user is required to use the existing soil samples to predict only the unvisited locations with very similar environmental conditions. In other words, only these existing samples that the similarities to the unvisited location are higher than 0.9 are used to calculate the value of the targeted property on this unvisited location. The spatial distribution of all the designed 38 additional samples in this uncertainty reduction stage is shown in Fig. 8.

\section{Results and discussion}

\subsection{Field sampling}

A total of 60 samples were collected finally (Fig. 9). Due to field conditions, only 3 of the designed samples were not collected at their designed locations. Fig. 9 compares the locations of samples designed with the locations actually collected. For these points deviated from their designed locations the environmental conditions of the replacement samples are similar to those of the designed locations.

\subsection{Validation and discussion}

The predicted soil property in this study is soil organic matter content of the top layer $(0-20 \mathrm{~cm})$. The regular sample set for validating map accuracy and the stratified random set for comparison between and uncertainty directed field sampling scheme and stratified random sampling were collected at the same time. The regular sample set includes 61 samples with the grid space of $10 \mathrm{~km}$ (Fig. 10), and these 61 samples were used for validation. The stratified random sample set includes 62 samples (Fig. 11) and these 62 samples were used for comparison.

For evaluating the performance of uncertainty directed sampling scheme, we created 60 sample sets by adding one additional sample into existing samples at each time. For this study area, we have 6 legacy samples. According to the order of the additional samples, add the first additional sample to the legacy sample set, and we got a sample set with 7 samples. Then, add the second sample to the new sample set, and we got a sample set with 8 samples. By iterating this procedure, we can get 60 sample sets after adding the 60th additional samples. Finally, based on these 60 sample sets, 60 soil organic matter property maps were produced using individual predictive soil mapping method (Zhu et al., 2015). Root Mean Square Error (RMSE) were calculated for each of the 60 soil property maps by comparing the predicted values with the observed values at the regular samples which fall into the predicted areas. This means that the number of validating points used for calculating the RMSE for each of the 60 soil property maps is different. This

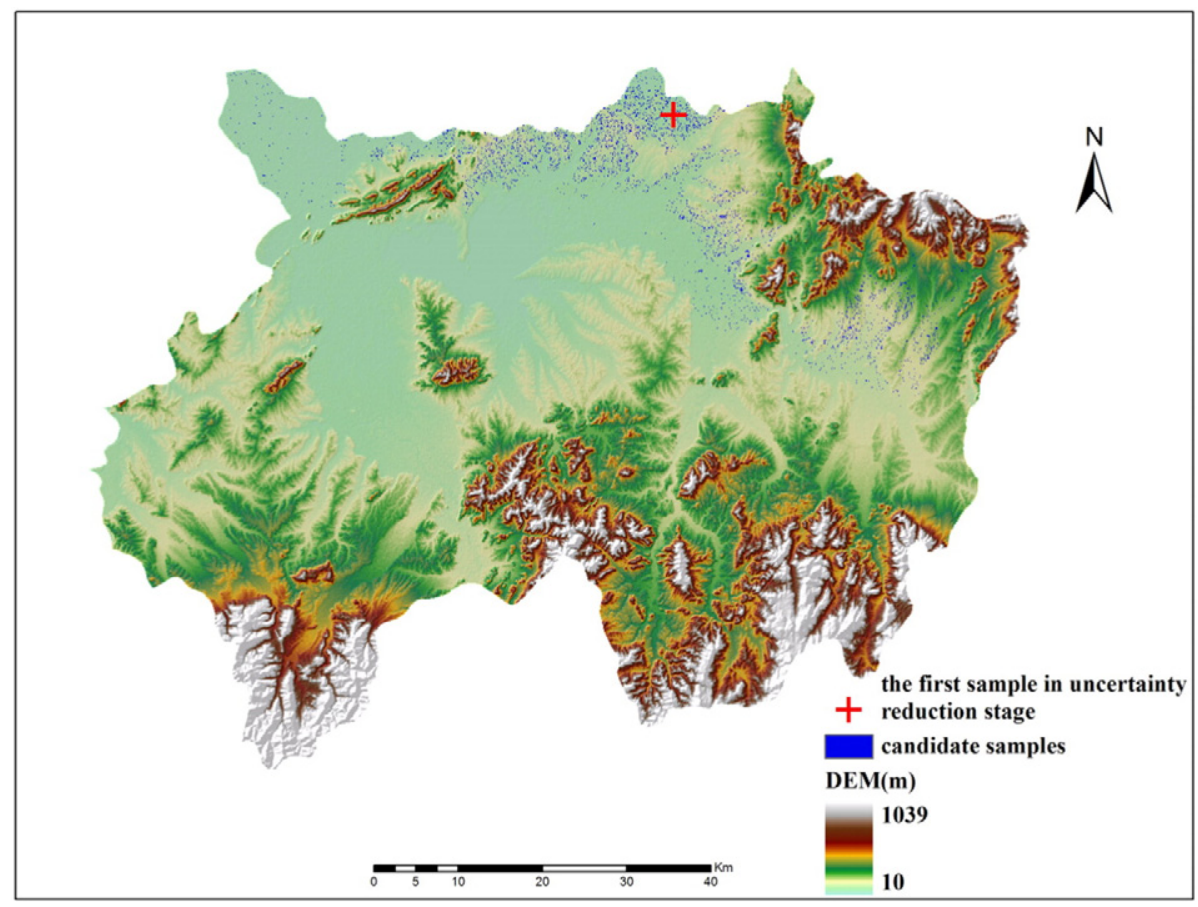

Fig. 7. The candidate samples and the first additional sample in the uncertainty reduction stage. 


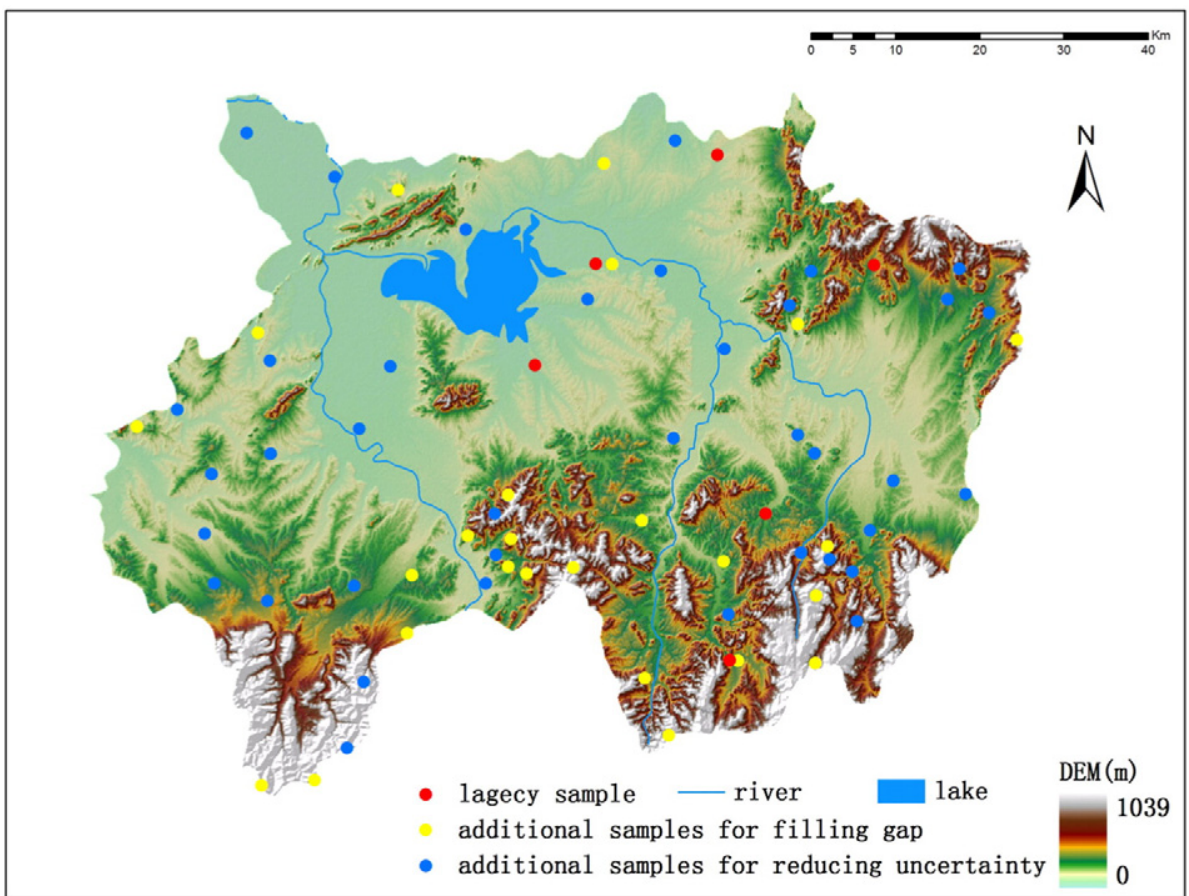

Fig. 8. The spatial distribution of additional samples designed for reducing overall uncertainty.

RMSE value is only used to evaluate the performance of uncertainty directed sampling scheme and is not used to examine how the RMSE change with respect to the increase of samples used in prediction because the number of validation samples which fall into the predicted areas is different for different predicted maps.

In order to compare the effectiveness of uncertainty directed sampling scheme with the stratified random sampling scheme, we also created 60 random sample sets using follow method: (1) Randomly choose a sample from the stratified random samples as the first additional sample, and then add this selected sample into the 6 legacy sample set to form a new random sample set with 7 samples; (2) Randomly choose another sample from the remaining stratified random samples and add this sample into the new sample set to form another new random sample set with 8 samples; (3) Iterate the second step 60 times, and we obtain 60 random sample sets. Based on these 60 random sample sets, another 60 soil property maps were produced using the same inference method proposed by Zhu et al. (2015).

A RMSE value was calculated for each of the 60 soil property maps by comparing the observed values with the predicted values based on the stratified random set at the locations of these validation samples

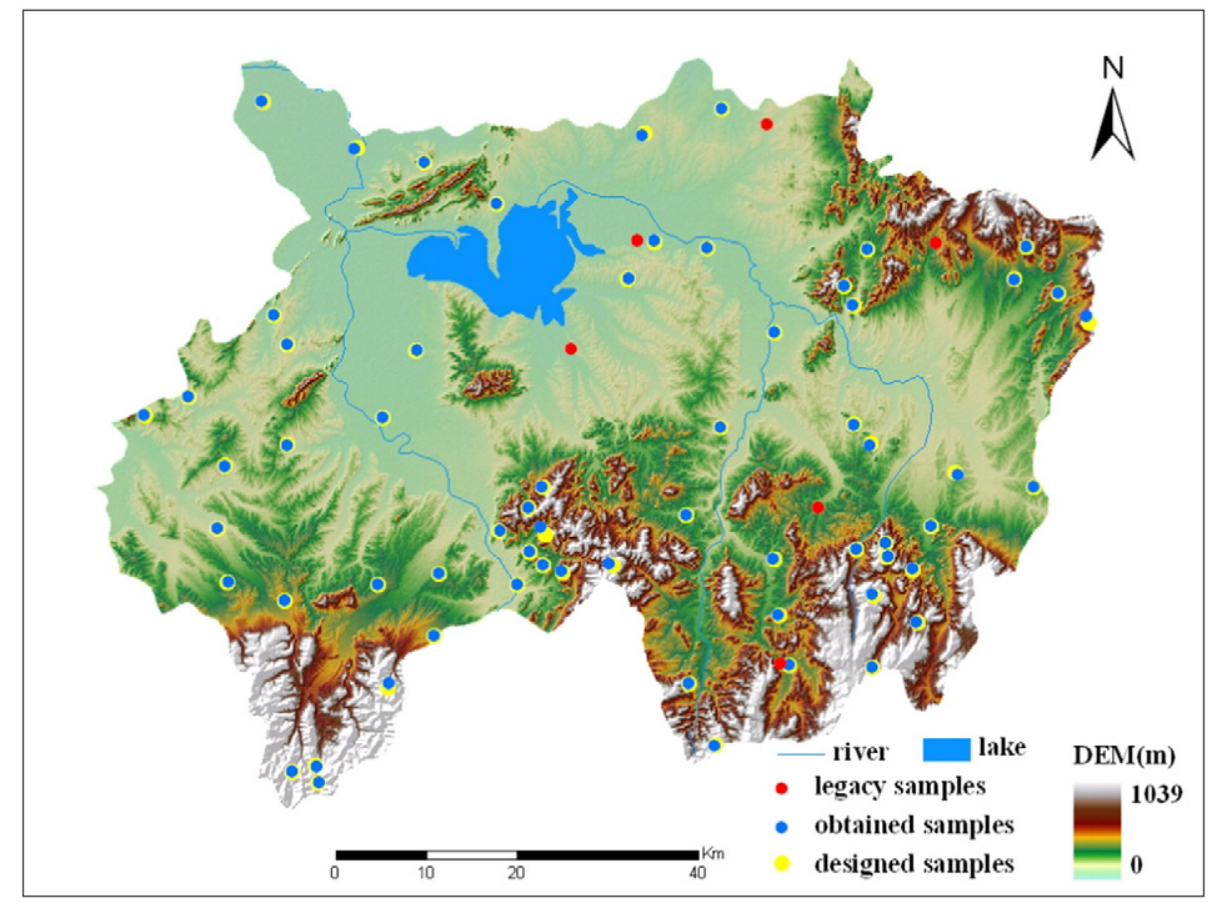

Fig. 9. The spatial distribution of obtained samples. 


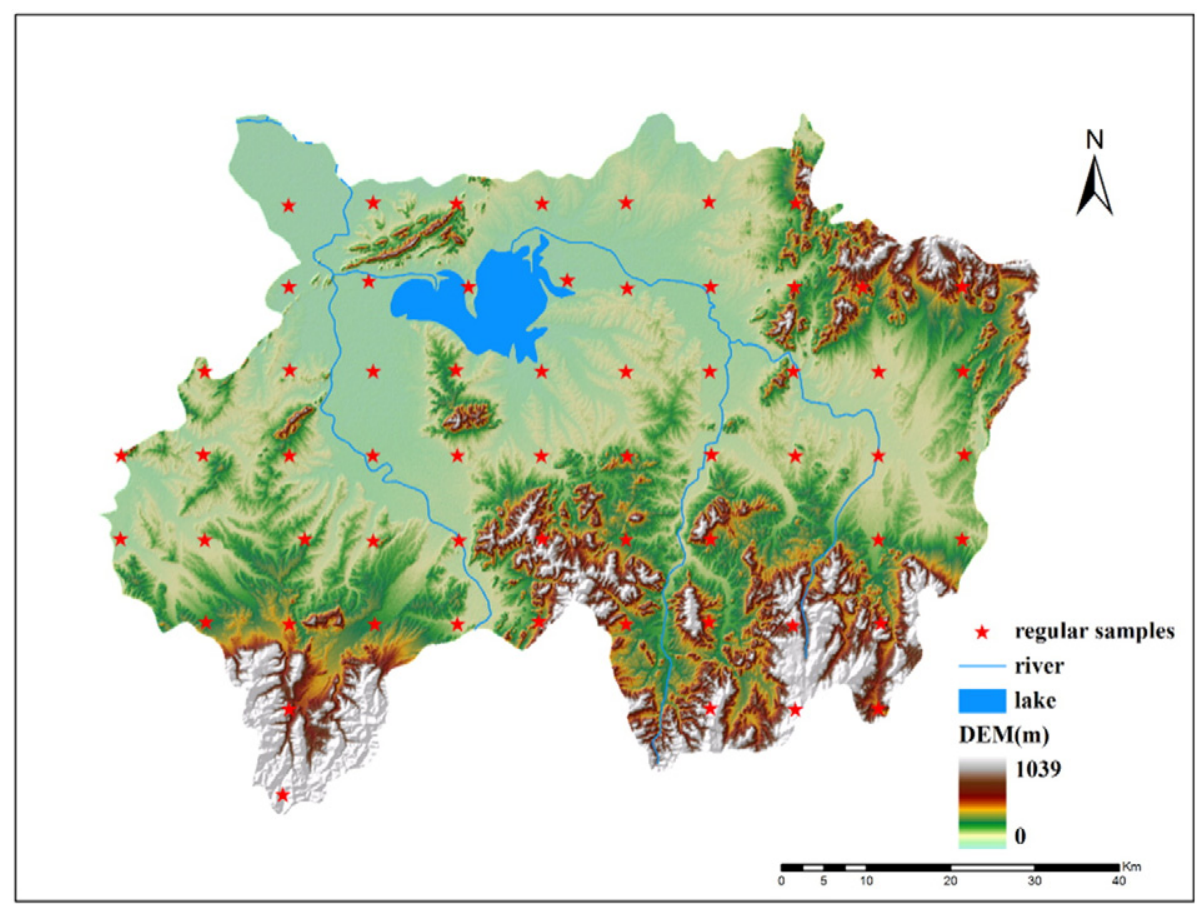

Fig. 10. The spatial distribution of regular samples.

which fall into the predicted areas. Because the order of the additional sample is random in the process of making the 60 random sample sets, the samples in each sample set could be different. Furthermore, when the order of additional samples is different, the produced soil maps are also different. In this paper, in order to validate the stratified random sampling scheme comprehensively, we repeat the above process of making the 60 random sample sets 5 times to obtain some representation of variety from the stratified random set.

We compared the effectiveness of the two sampling schemes (uncertainty directed sampling scheme and 5 stratified random sampling schemes) in two aspects: the expansion of predicted area (Fig. 12) and the accuracy of soil maps (RMSE) (Fig. 13).

The X coordinate of Fig 12 is the order of additional samples, and the $\mathrm{Y}$ coordinate is the predicted area which is the number of the predicted grids. The red line represents the variation of predicted area for uncertainty directed sample scheme and other five lines represent the predicted area for stratified random sample schemes. For the uncertainty directed samples, as we expected, the predicted area extended quickly and the gaps in the generated soil map were quickly filled when more samples are successively added. The predicted area reaches a plateau

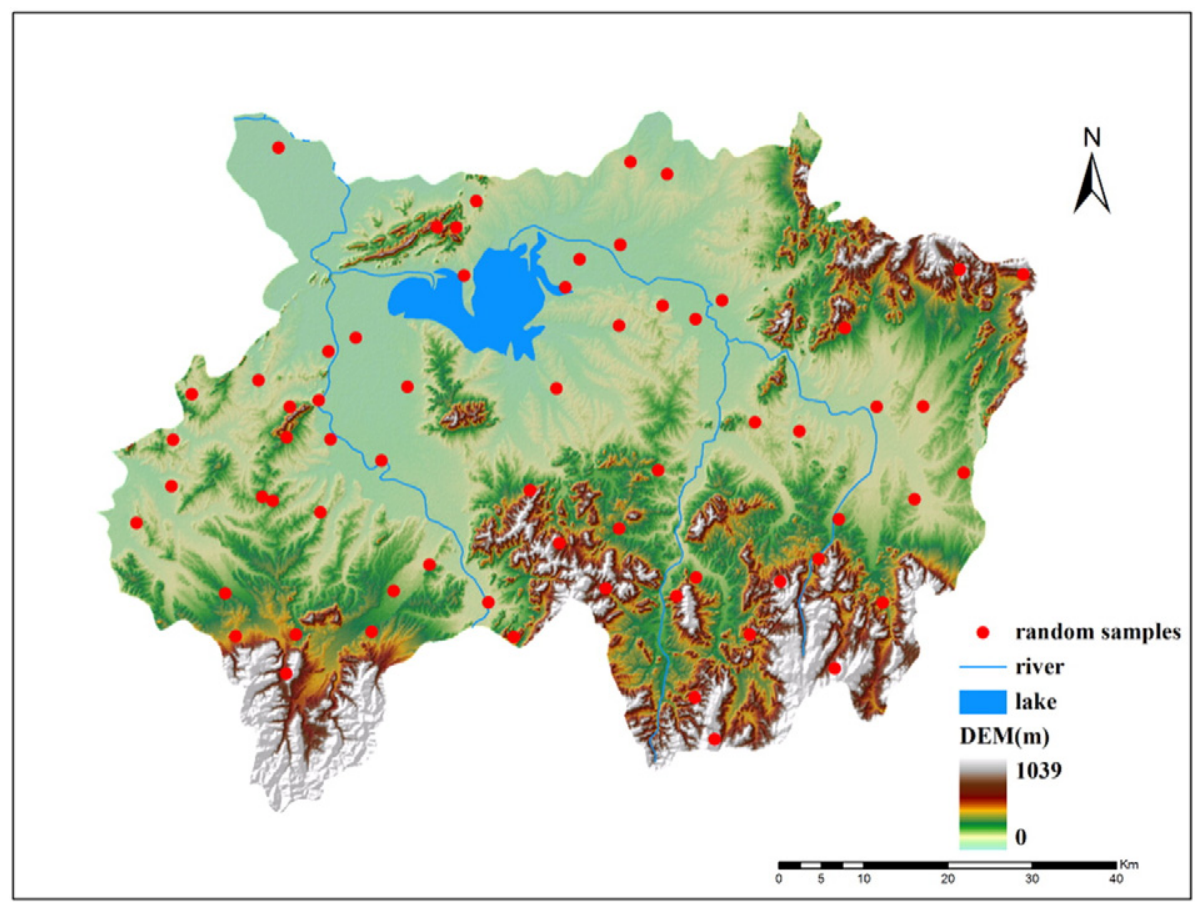

Fig. 11. The spatial distribution of stratified random samples. 


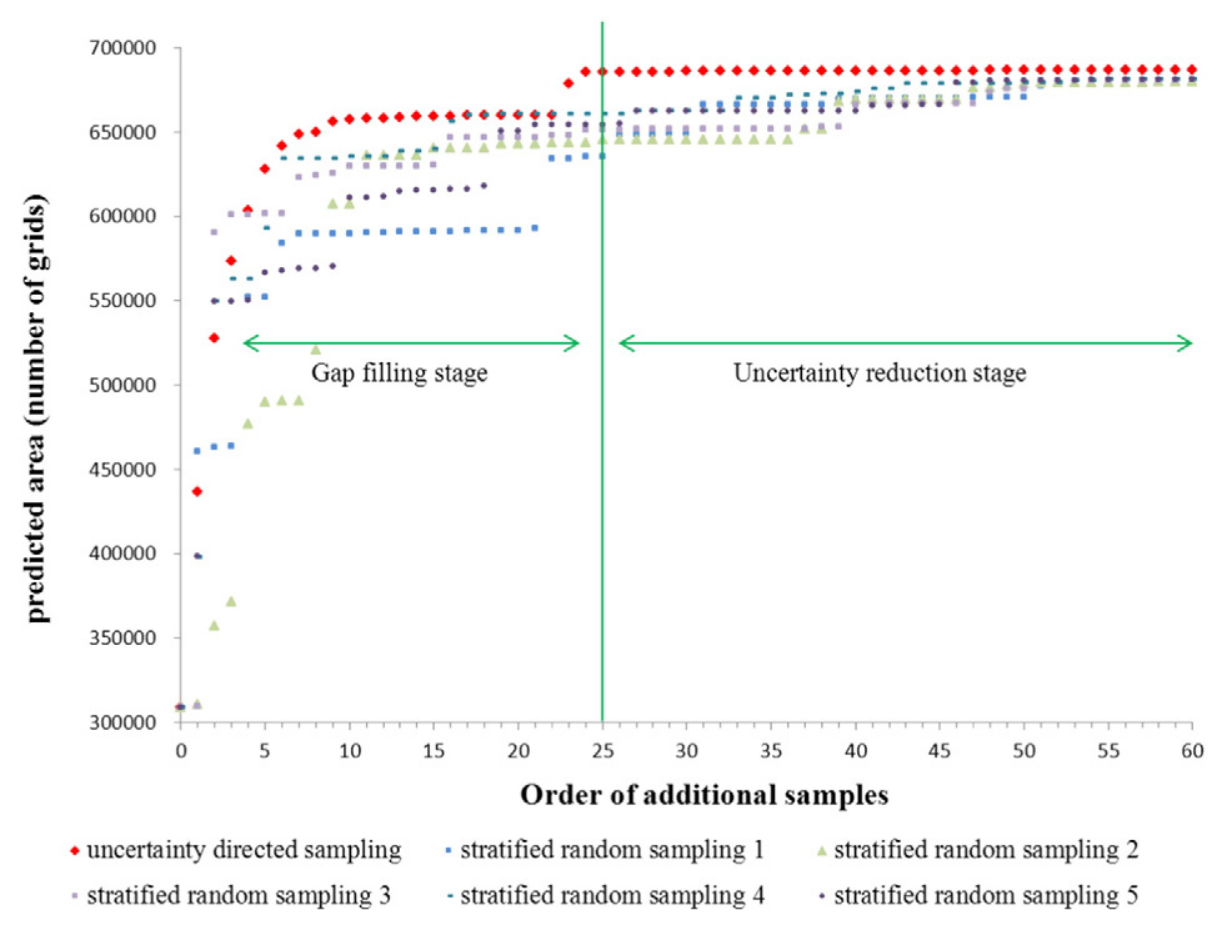

Fig. 12. Variation of predicted area with additional samples.

at the end of the filing gap stage, which demonstrates that the predicted area has almost covered the whole study area. Moreover, the incremental predicted area is larger for the first few additional samples than the later samples, which shows that the method was able to effectively locate the additional samples for covering the area. Comparing with the stratified random sampling schemes, the increment in area coverage for the first few samples based on the uncertainty directed approach is larger. Another important advantage of the uncertainty directed approach is that predicted area increases continuously and eventually stabilized. This can be used to determine the number of points needed for covering the entire area even before field sampling starts.

The X coordinate of Fig. 13 is also the order of additional samples and the $Y$ coordinate is RMSE. The red line represents the RMSE calculated based on the 60 soil organic matter maps produced using the 60

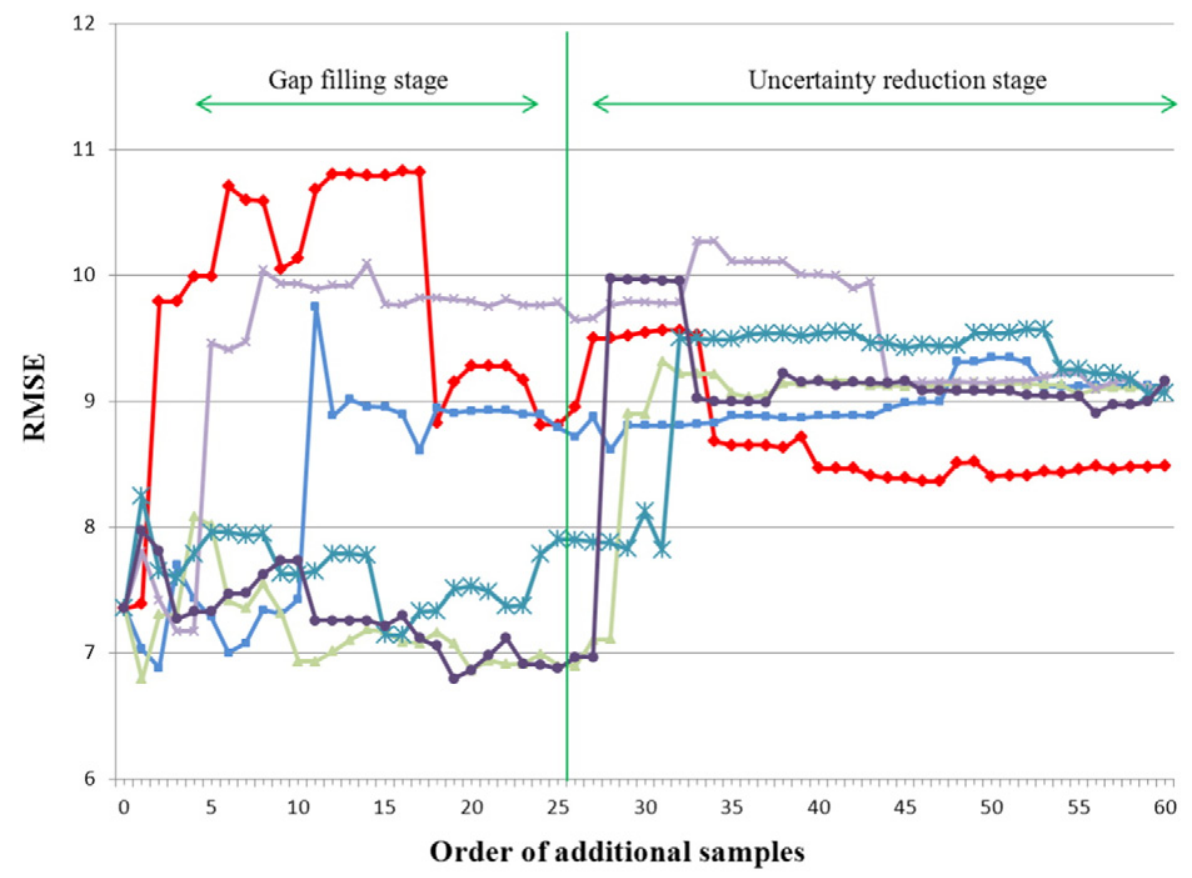

$\longrightarrow$ uncertainty directed sampling $\longrightarrow$ stratified random sampling $1 \multimap$ stratified random sampling 2
- stratified random sampling $3 \rightarrow$ stratified random sampling $4 \rightarrow$ stratified random sampling 5

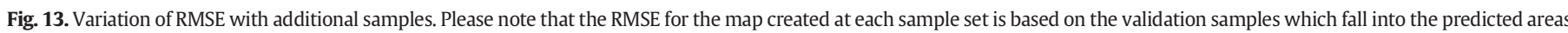
for that map. 


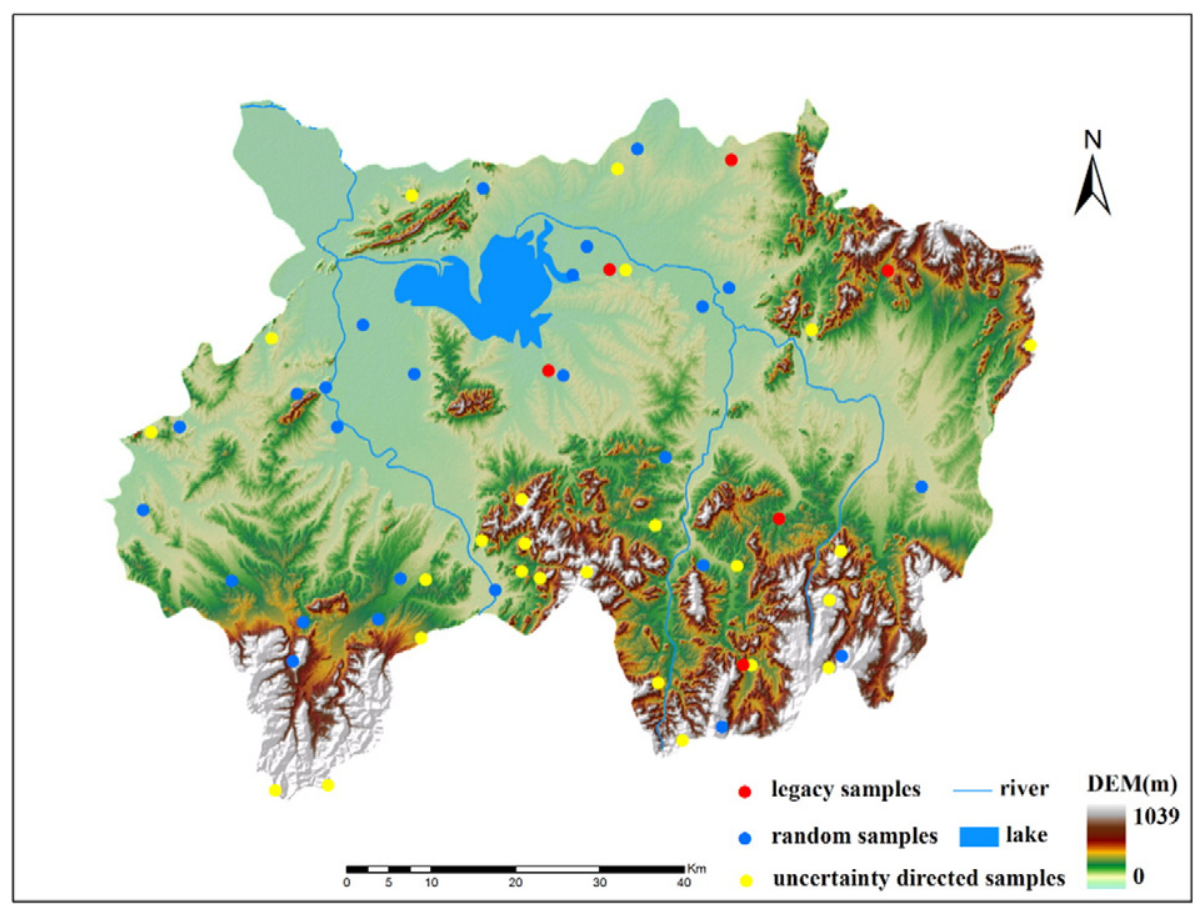

Fig. 14. The spatial distribution of the first 25 uncertainty directed samples and the first 25 random samples (take one of the 5 random sample sets as an example).

uncertainty directed sample sets and the other 5 RMSE lines represent these based on the five stratified random trials.

During the gap filling stage, the RMSE of the soil maps produced using uncertainty directed samples is higher than the RMSE of soil maps produced using stratified random samples. This is because during this stage uncertainty directed sampling scheme is optimized to cover the size of predicted area. For example, from Fig. 14, we can see that, in order to extend the predicted area as much as possible most of the uncertainty directed samples were located in the southern mountain area where the area coverage of each sample is small and more samples are needed to represent the complex environmental conditions. However, unlike uncertainty directed sampling scheme, most of the stratified random samples were located in the middle and north plain area because the number of the samples is related to the area of the parent material and the parent material of plain accounts for most of the study area. Meanwhile, most of the regular validation samples are also in this plain area. Therefore, the stratified random samples represent regular validation samples better than uncertainty directed samples, which results in the RMSE of stratified random sample is relatively low.

In the uncertainty reduction stage, the objective for uncertainty directed sampling is to reduce the overall prediction uncertainty for improving the accuracy. From Fig. 13, we can see that the RMSE for uncertainty directed sampling scheme declines slowly during the uncertainty reduction stage. Starting from the 34th additional sample, RMSE of uncertainty directed sampling scheme is lower than all of the trials based on the stratified random sampling scheme. In other words, from the 34th sample on, the accuracy of soil organic matter maps based on the uncertainty directed samples is higher than the maps based on stratified random samples. It should also be noted in Fig. 13 that the increase in RMSE for all sampling schemes when switched from the gap filling stage to the uncertainty reduction stage is related to the fact that during the gap filling stage only the validation samples which fall into the predicted areas were used for computing the RMSE but during the uncertainty reduction stage all validation samples were used to calculate the RMSE. It is expected that with more validation samples the RMSE is higher.

\section{Conclusions}

This paper presented an effective and stepwise method to design õadditional samples for digital soil mapping based on prediction uncertainty. This sampling scheme not only integrates the sparse and ad hoc legacy samples with additional new samples effectively, but also provides the order of additional samples based on their respective contributions to area coverage and accuracy improvement. The order of the additional samples allows us to effectively use the limited sampling resources. The case study in XuanCheng study area demonstrated that this uncertainty directed sampling scheme is more effective than stratified sampling scheme. In the gap filling stage, the uncertainty directed samples cover the study area more quickly. In the uncertainty reduction stage, the RMSE reduces quickly and it is lower than these based on the stratified random samples. In a word, the proposed method is effective in locating as few additional samples as possible to extend predicted area and to maximize the reduction in uncertainty.

\section{Acknowledgments}

This study was supported by the National Natural Science Foundation of China (No. 41431177; 41471178), the Natural Science Research Program of Jiangsu (No. 14KJA170001), the National Basic Research Program of China (No. 2015CB954102), the National Key Technology Innovation Project for Water Pollution Control and Remediation (No. 2013ZX07103006), the National Science and Technology Support Program (No. 2013BAC08B03-4), and IGSNRR Director's Research Fund for Innovation (No. Y3W30020YZ). The first author appreciates the support from the members of 2415 Room of IGSNRR for in field sampling. The support received by A-Xing Zhu through the Vilas Associate Award, the Hammel Faculty Fellow, and the Manasse Chair Professorship from the University of WisconsinMadison and through the "One-Thousand Talents" Program of China is greatly appreciated. The support to Chengzhi Qin from the National Natural Science Foundation of China through its outstanding young scientist progam under the contract number 41422109 is also greatly appreciated. 


\section{References}

Beaudoin, A., Bernier, P.Y., Guindon, L., Villemaire, P., Guo, X.J., Stinson, G., Bergeron, T. Magnussen, S., Hall, R.J., 2014. Mapping attributes of Canada's forests at moderate resolution through kNN and MODIS imagery. Can. J. For. Res. 44, 521-532.

Behrens, T., Zhu, A.X., Schmidt, K., Scholten, T., 2010. Multi-scale digital terrain analysis and feature selection for digital soil mapping. Geoderma 155, 175-185.

Brus, D.J., de Gruijter, J.J., 1997. Random sampling or geostatistical modelling? Choosing between design-based and model-based sampling strategies for soil. Geoderma 80, $1-59$

Brus, D.J., Heuvelink, G.B.M., 2007. Optimization of sample patterns for universal kriging of environmental variables. Geoderma 138, 86-95.

Brus, D.J., de Gruijter, J.J., van Groenigen, J.W., 2006. Designing spatial coverage samples using the k-means clustering algorithm. In: Lagacherie, P., McBratney, A.B., Voltz, M. (Eds.), Digital Soil Mapping: An Introductory Perspective. Elsevier, New York, pp. 183-192.

Feng, X., Liu, G., Chen, J.M., Chen, M., Liu, J., Ju, W.M., Sun, R., Zhou, W., 2007. Net primary productivity of China's terrestrial ecosystems from a process model drive by remote sensing. J. Environ. Manag. 85, 563-573.

Goovaerts, P., 1999. Geostatistics in soil science: state-of-the-art and perspectives. Geoderma 89, 1-45.

Hudson, B.D., 1992. The soil survey as paradigm-based science. Soil Sci. Soc. Am. J. 56, $836-841$.

Isaaks, E.H., Srivastava, R.M., 1989. An Introduction to Applied Geostatistics. Oxford University Press, New York (561 pp.).

Jenny, H., 1941. Factors of Soil Formation - A System of Quantitative Pedology. Dover Publications, New York (281 pp.).

Kidd, D., Malone, B., McBratney, A., Minasny, B., Webb, M., 2015. Operational sampling challenges to digital soil mapping in Tasmania, Australia. Geoderma Reg. 4, 1-10.

Liu, F., Geng, X.Y., Zhu, A.X., Fraser, W., Arnie, W., 2012. Soil texture mapping over low relief areas using land surface feedback dynamic patterns extracted from MODIS. Geoderma 171-172, 44-52.

Mansuy, N., Thiffault, E., Pare, D., Bernier, P., Guindon, L., Villemaire, P., Poirier, V. Beaudoin, A., 2014. Digital mapping of soil properties in Canadian managed forests at $250 \mathrm{~m}$ of resolution using the k-nearst neighbor method. Geoderma 235-236, 59-73.

McBratney, A.B., Mendonca Santos, M.L., Minasny, B., 2003. On digital soil mapping. Geoderma 117, 3-52.

Minasny, B., McBratney, A.B., 2006. A conditioned Latin hypercube method for sampling in the presence of ancillary information. Comput. Geosci. 32 (9), 1378-1388.

Nield, S.J., Boettinger, J.L., Ramsey, R.D., 2007. Digitally mapping gypsic and natric soil areas using Landsat ETM data. Soil Sci. Soc. Am. J. 71, 245-252.

Pechenizkiy, M., Puuronen, S., Tsymbal, A., 2003. Feature extraction for classification in knowledge discovery systems. Proc. 7th Int. Conf. On Knowledge-Based IntelligentInformation \& Engineering Systems, Berlin, pp, pp. 526-532.

Qi, F., Zhu, A.X., Harrower, M., Burt, J.E., 2007. Fuzzy soil mapping based on prototype category theory. Geoderma $130,774-787$

Qin, C.Z., Zhu, A.X., Pei, T., Li, B.L., Zhou, C.H., Yang, L., 2007. An adaptive approach to selecting a flow-partition exponent for a multiple-flow-direction algorithm. Int. J. Geogr. Inf. Sci. 21 (4), 443-458.

Qin, C.Z., Zhu, A.X., Qiu, W.L., Lu, Y.J., Li, B.L., Pei, T., 2012. Mapping soil organic matter in small low-relief catchments using fuzzy slop position information. Geoderma 171$172,64-74$.

Ramadan, Z., Song, X.H., Hopke, P.K., Johnson, M.J., Scow, K.M., 2001. Variable selection in classification of environmental soil samples for partial least square and neural network models. Anal. Chim. Acta 446, 233-244.

Reza Pahlavan Rada, M., Toomanian, N., Khormali, F., W. Brungard, W., Komaki, C.B. Bogaert, P., 2014. Updating soil survey maps using random forest and conditioned Latin hypercube sampling in the loess derived soils of northern Iran. Geoderma 232-234, 97-106.
Samuel-Rose, A., Heuvelink, G.B.M., Vasques, G.M., Anjos, L.H.C., 2015. Do more detailed environmental covariates deliver more accurte soil map? Geoderma 243-244, 214-227.

Sheather, S.J., Jones, M.C., 1991. A reliable data-based bandwidth selection method for kernel density estimation. J. R. Stat. Soc. 53 (3), 683-690.

Shi, X., Zhu, A.X., Wang, R., Fred, P., 2005. Fuzzy representations special terrain feature using a similarity-based approach. In: Vincent, B.R. (Ed.), Fuzzy modeling with spatial information for geographic problems. Springer, New York, pp. 233-251.

Shi, X.Z., Yu, D.S., Gao, P., Wang, H.J., Sun, W.X., Zhao, Y.C., Gong, Z.T., 2007. Soil information system of China (SISChina) and its application. Soil 39 (3), 329-333 (In Chinese with English abstract)

Silverman, B.W., 1986. Density Estimation for Statistics and Data Analysis. Chapman and Hall, London, United Kingdom (175 pp.).

Sun, X.L., Zhao, Y.G., Qin, C.Z., Li, D.C., Zhao, L., Zhang, G.L., 2008. Effects of DEM resolution on multi-factor linear soil-landscape models and their application in predictive soil mapping. Acta Pedol. Sin. 45 (5), 971-977 (In Chinese with English abstract).

Taghizadeh-Mehrjardi, R., Minasny, B., Sarmadian, F., Malone, B.P., 2014. Digital mapping of soil salinity in Ardakan region, central Iran. Geoderma 213, 15-28.

Van Groenigen, J.W., 2000. The influence of variogram parameters on optimal sampling schemes for mapping by kriging. Geoderma 97, 223-236.

Van Groenigen, J.W., Siderius, W., Stein, A., 1999. Constrained optimization of soil sampling for minimization of the kringing variance. Geoderma 87, 239-259.

Walvoort, D.J.J., Brus, D.J., de Gruijter, J.J., 2010. An R package for spatial coverage sampling and ranmdom sampling from compact geographical strata by k-means. Comput. Geosci. 36 (10), 1261-1267.

Worsham, L., Markewitz, D., Nibbelink, N.P., West, L.T., 2012. A comparison of three field sampling methods to estimate soil carbon content. For. Sci. 58, 513-522.

Yang, L., Jiao, Y., Fahmy, S., Zhu, A.X., Hann, S., Burt, J.E., Qi, F., 2011. Updating conventional soil maps through digital soil mapping. Soil Sci. Soc. Am. J. 75 (3), 1044-1053.

Yang, L., Zhu, A.X., Qi, F., Qin, C.Z., Li, B.L., Pei, T., 2013. An integrative hierarchical sampling strategy for spatial sampling and its application in digital soil mapping. Int. J. Geogr. Inf. Sci. 27 (1), 1-23.

Yang, L., Zhu, A.X., Zhao, Y.G., Li, D.C., Zhang, G.L., Zhang, S.J., Band, L.E., 2015. Regional Soil Mapping Using Multi-grade Representative Sampling and a Fuzzy Membership Based Mapping Approach. Pedosphere (in press).

Yu, D.S., Shi, XZ W Wang, H.J., Sun, W X., Chen, J.M. Liu, Q.H, Zhao, Y.C. 2007. Regional patterns of soil organic carbon stocks in China. J. Environ. Manag. 85, 680-689.

Yu, G.R., He, H.L., Liu, X.A., Niu, D., 2004. Study on spatialization technology of terrestria eco-information in China (I): the approach of spatialization in meteorology/climate information. J. Nat. Res. 19 (4), 537-544 (In Chinese with English abstract).

Zhao, L., Zhao, Y.G., Li, D.C., Sun, X.L., Zhang, G.L., 2007. Digital soil mapping by extracting quantitative relationships between soil properties and terrain factors based on fuzzy set theory. Acta Pedol. Sin. 44 (6), 961-967 (In Chinese with English abstract).

Zhu, A.X., 1997. Measuring uncertainty in class assignment for natural resource maps using a similarity model. Photogramm. Eng. Remote Sens. 63, 1195-1202.

Zhu, A.X., Band, L.E., 1994. A knowledge-based approach to data integration for soil mapping. Can. J. Remote. Sens. 20, 408-418.

Zhu, A.X., Band, L.E., Dutton, B., Nimlos, T.J., 1996. Automated soil inference under fuzzy logic. Ecol. Model. 90, 123-145.

Zhu, A.X., Yang, L., Li, B.L., Qin, C.Z., English, E., Burt, J.E., Zhou, C.H., 2008. Purposive sampling for digital soil mapping for areas with limited data. In: Hartemink, A.E., McBratney, A.B., Mendonca Santos, M.L. (Eds.), Digital Soil Mapping with Limited Data. Springer-Verlag, New York, pp. 233-245.

Zhu, A.X., Qi, F., Moore, A., Burt, J.E., 2010(a)a. Prediction of soil properties using fuzzy membership values. Geoderma 158, 199-206.

Zhu, A.X., Liu, F., Li, B.L., Pei, T., Qin, C.Z., Liu, G.H., Wang, Y.J., Chen, Y.N., Ma, X.W., Qi, F., Zhou, C.H., 2010(b)b. Differentiation of soil conditions over flat areas using land surface feedback dynamic patterns extracted from MODIS. Soil Sci. Soc. Am. J. 74, 861-869.

Zhu, A.X., Liu, J., Du, F., Zhang, S.J., Qin, C.Z., Burt, J., Behrens, T., Scholten, T., 2015. Predictive soil mapping with limited sample data. Eur. J. Soil Sci. 66 (3), 535-547. 